\title{
BMJ Open Identifying patient-important outcomes in medication-assisted treatment for opioid use disorder patients: a systematic review protocol
}

\author{
Nitika Sanger, ${ }^{1,2}$ Hamnah Shahid, ${ }^{2,3}$ Brittany B Dennis,, ${ }^{4,5}$ Jackie Hudson, ${ }^{2}$ \\ David Marsh, ${ }^{6,7}$ Stephanie Sanger, ${ }^{8}$ Andrew Worster, ${ }^{5,9}$ Rand Teed, ${ }^{10}$ \\ Launette Rieb, ${ }^{11}$ Peter Tugwell, ${ }^{12,13,14,15}$ Brian Hutton, ${ }^{12,16}$ Beverley Shea, ${ }^{12,13,14}$ \\ Dorcas Beaton, ${ }^{17,18}$ Kimberly Corace, ${ }^{19,20}$ Danielle Rice, ${ }^{12}$ Lara Maxwell, ${ }^{12}$ \\ M Constantine Samaan, ${ }^{21}$ Russell J de Souza, ${ }^{22}$ Lehana Thabane, ${ }^{22,23}$ \\ Zainab Samaan ${ }^{2,22}$
}

To cite: Sanger N, Shahid $\mathrm{H}$ Dennis BB, et al. Identifying patient-important outcomes in medication-assisted treatment for opioid use disorder patients: a systematic review protocol. BMJ Open 2018;8:e25059. doi:10.1136/ bmjopen-2018-025059

- Prepublication history for this paper is available online. To view these files, please visit the journal online (http://dx.doi org/10.1136/bmjopen-2018025059).

Received 28 June 2018 Revised 22 October 2018 Accepted 5 November 2018

Check for updates

(c) Author(s) (or their employer(s)) 2018. Re-use permitted under CC BY-NC. No commercial re-use. See rights and permissions. Published by BMJ.

For numbered affiliations see end of article.

Correspondence to

Dr Zainab Samaan;

samaanz@mcmaster.ca

\section{ABSTRACT}

Introduction Illicit opioid use has become a national crisis in Canada, with over 65000 people seeking treatment for opioid use disorder (OUD) in Ontario and British Columbia alone. Medication-assisted treatment (MAT) is a common treatment for OUD. There is substantial variability in treatment outcomes used to evaluate effectiveness of MAT, making it difficult to establish clinically and scientifically relevant treatment effect. Furthermore, patients are often excluded from the process of determining these outcomes. The primary objective of this review is to examine outcomes currently used to measure MAT effectiveness and to identify patient-relevant outcomes to enhance effectiveness of treatment options. This review refers to patient-important outcomes as those outcomes patients consider important to or markers of treatment success.

Methods and analysis MEDLINE, EMBASE, PsycINFO, Cumulative Index to Nursing and Allied Health Literature, Web of Science, Cochrane Library, Cochrane Clinical Trials Registry, National Institutes for Health Clinical Trials Registry and WHO International Clinical Trials Registry Platform databases will be searched. We will search databases from inception to the date the search is ran. Studies of interest include those evaluating the effectiveness of MAT for patients with OUD, with or without consultation with patients regarding what they consider to be important as an indicator of treatment success. Results will be analysed using thematic analysis and qualitative analysis where possible. This will result in comprehensive synthesis of all outcomes and measures found related to OUD treatment effectiveness.

Ethics and dissemination We are collaborating with Canadian Addiction Treatment Centres which provide MAT to patients with OUD who will participate in disseminating study results. Dissemination strategies will involve sharing study results through workshops, presentations, peer-reviewed publications, study reports, community presentations and resources in primary care settings.

PROSPERO registration number CRD42018095553.

\section{Strengths and limitations of this study}

This will be the first systematic review examining any patient-important outcomes that have been reported relating to the effectiveness of medication-assisted treatment (MAT) among people with opioid use disorder

- Additional qualitative distributions will be examined based on study age group (adolescents, adults), sex, country and type of MAT.

- There may be variability in how treatment outcomes are measured in studies, which may pose a challenge in the qualitative synthesis and thematic analysis.

- There may be a paucity in patient-important outcomes reported in the published clinical trials testing the effectiveness of MAT for opioid use disorder.

\section{INTRODUCTION}

\section{Rationale}

Illicit opioid use has become a crisis in Canada, with a number of people being diagnosed with opioid use disorder (OUD)..$^{12}$ Between 2015 and 2016, approximately one in seven people in Ontario filled an opioid prescription, which the Canadian Guideline for Opioid Therapy and Chronic Non-Cancer Pain has reported are associated with a $5.5 \%$ risk of addiction. ${ }^{3}$ Canada in particular has seen a $203 \%$ increase in usage between 2000 and 2010. ${ }^{4}$ Such an increase in opioid use is concerning, as opioid poisonings have resulted in more than 16 hospitalisations a day in Canada. ${ }^{5}$ From 2007-2008 to 20142015 , the crude rate of hospitalisations due to opioid poisonings increased by over $30 \%{ }^{6}$ According to the national report on opioid-related deaths, there were 3987 opioid-related 
fatalities in Canada in 2017 alone. ${ }^{7}$ Based on this count, the apparent opioid-related death rate in Canada was 8.8 per 100000 that year. ${ }^{7}$ Considering the substantial health risk opioids pose in Canada, it is vital to identify effective ways of treating OUD and enhancing patients' recovery and treatment outcomes.

Currently, there are over 65000 patients in Ontario and British Columbia combined seeking pharmacological treatment for OUD, most commonly via methadone maintenance treatment (MMT) ${ }^{8-10}$ MMT is the oldest and one of the most commonly used treatment for OUD. However, it is one of several medication-assisted treatment (MAT) options available. ${ }^{11} 12$ MAT range from a variety of treatments available including opioid agonists such as methadone and buprenorphine/naloxone to opioid antagonists such as naltrexone. ${ }^{12}{ }^{13}$ Recent guidelines indicate there is little consistent evidence to evaluate the effectiveness of MATs. ${ }^{14}$ Reviews evaluating MAT effectiveness have found great variability in outcomes between studies, ${ }^{15-17}$ making it difficult to establish a real treatment effect. Each study measures a different set of treatment outcomes that define success in arbitrary or convenient terms. This is a substantial limitation in addiction research that must be overcome to reach a consensus on which treatment outcome domains should be the goals, how those outcome domains should be measured and what works for opioid addiction management. Who decides on what outcome is important? As a healthcare provider, one might consider abstinence the ultimate goal of addiction treatment, while a patient may desire control over their substance use or symptom management to be the goal of treatment. Additionally, researchers must consider how trial design is influenced by selection of outcomes. If the outcome for such trials was reduced criminal activity, reduced incidence of infectious diseases, reduced homelessness or other social advantage, the intervention may be helpful for only certain groups of patients. Regardless, without the identification of a measurable treatment outcome that has an impact and significance to patients, services and the population as a whole, all the investment in trials will result in inadequate and inconsistent 'effectiveness' with limited, if any, external validity. There needs to be a set of established MAT outcomes, so that healthcare providers and their patients, pharmacotherapeutics developers, policy makers, researchers, budget holders and service users are able to evaluate the true effectiveness of these therapies.

While establishing empirical outcomes for MATs is crucial, it is also vital to understand which treatment outcomes the patients consider important. Clinicians, researchers, patients and other stake holders may have different views in in this regard, and thus a comprehensive assessment of what outcomes should be considered especially taking the patients' perspective must be included. Unfortunately, patient-centred outcomes are often neglected in effectiveness trials, an issue especially marked within the addiction literature. A recent systematic review evaluating the effectiveness of different MATs for OUD suggests the literature has no consistent definition of a 'successful' addiction treatment outcome, and thus most trials assessing therapy effectiveness use a variety of end points. ${ }^{18}$ Treatment attrition, continued opioid use, criminal activities, quality of life and economic costs are among the long list of outcomes used across trials aimed to establish treatment effectiveness in the OUD literature. There are also very few validated tools such as the Addiction Severity Index ${ }^{19}$ and the Maudsley Addiction Profile ${ }^{20}$ which measure health improvement across various domains. The variation in the selection of outcomes and the many different ways of defining and measuring those outcomes call for a need to determine a set of outcomes that are patient informed in addiction treatment. To our knowledge, an evaluation of patients' treatment goals is often embedded in larger studies, and thus has not been investigated in a systematic review.

\section{Objectives}

The purpose of this systematic review and meta-analysis is to investigate the outcomes currently used to measure MAT effectiveness and to identify patient-relevant outcomes to enhance the effectiveness of current treatment options.

Specifically, our objectives are to:

1. Summarise all treatment outcome domains used in clinical trials to measure MAT effectiveness for OUD, and how these outcomes are assessed.

2. Summarise the observational and qualitative literature reporting patient-identified important treatment outcome domains (goals and markers that patient identify as a measure of success fort themselves) for OUD.

\section{METHODS AND ANALYSIS}

This protocol was reported using the Preferred Reporting Items for Systematic Reviews and Meta-analyses Protocols statement. $^{21}$

\section{Eligibility criteria}

The first search strategy will include randomised controlled trials (RCTs) examining any and all treatment outcomes of OUD MAT. There will be no restriction on age of the study participants, as described above. Studies will be excluded if they are animal studies or are incomplete studies (ie, internal pilot, preliminary reports). Furthermore, if multiple studies have been published using the same registration number, only the most recent will be included.

The second search strategy will include observational studies (excluding case studies, case series and reviews) and qualitative research, such as, but not limited to, interviews and focus groups, that examine what outcomes are important to patients in MATs regarding treatment success. Similar to the first strategy, there will be no restriction on age. 
Table 1 Search strategy for objective 1

Database
MEDLINE $\begin{aligned} & \text { 1. methadone/th [Therapy] } \\ & \text { 2. opioid substitution treatment/ae, mo [adverse effects, mortality] } \\ & \text { 3. substance-Related Disorders/de, ep, th [Drug Effects, Epidemiology, Therapy] } \\ & \text { 4. Opiate Substitution Treatment/or Buprenorphine/or Opioid-Related Disorders/or Heroin } \\ & \text { Dependence/or Substance Withdrawal Syndrome/or Narcotic Antagonists } \\ & \text { 5. exp Buprenorphine, Naloxone Drug Combination/ } \\ & \text { 6. Naltrexone/ae, ag, ai, tu [Adverse Effects, Agonists, Antagonists \& Inhibitors, Therapeutic Use] } \\ & \text { 7. Substance Abuse Treatment Centers/or Substance Abuse, Intravenous/or Heroin/or Heroin } \\ & \text { Dependence/or Opioid-Related Disorders/or Randomized Controlled Trials/or Methadone } \\ & \text { 8. } 1 \text { OR } 2 \text { OR } 4 \text { OR } 5 \text { OR } 6 \text { OR } 7 \\ & \text { 9. } 3 \text { AND } 8 \\ & \text { 10. limit } 9 \text { to humans }\end{aligned}$

\section{Outcomes and prioritisation}

The first objective will assess which outcomes are currently being used in RCTs to measure the effectiveness of MAT. For example, these outcomes may include continued opioid use, other substance use, physical adverse events (ie, emergency room visits, hospitalisations), social adversity (ie, criminal activity, unemployment, marital discord) and mortality.

The second objective will be based on observational and qualitative studies and will determine which outcomes patients have indicated are important for them while receiving MAT. These outcomes may include, for example, securing employment, reducing intravenous drug use, regaining custody of children and reducing withdrawal symptoms.

\section{Information sources}

We will be searching the following databases for this review: MEDLINE, EMBASE, PsycINFO, Cumulative
Index to Nursing and Allied Health Literature, Web of Science, Cochrane Library, Cochrane Clinical Trials Registry, the National Institutes for Health Clinical Trials Registry and the WHO International Clinical Trials Registry Platform. Articles will be obtained using comprehensive search strategies (tables 1 and 2). We will search databases from inception to the date the search is ran. We have created the two searches to reflect the most encompassing terms thus far but will include the final strategies for all databases in the full review.

The first strategy (table 1) includes search terms related to MAT and RCT, and their medical subject headings in varying combinations, while the second strategy (table 2) includes terms related to MAT and patient-important outcomes in various combinations. Additionally, we will manually search through reference lists of articles that pass the initial abstract screening for any relevant articles the search strategy may not have captured. An

Table 2 Search strategy for objective 2

\begin{tabular}{|c|c|}
\hline Database & Search strategy \\
\hline MEDLINE & 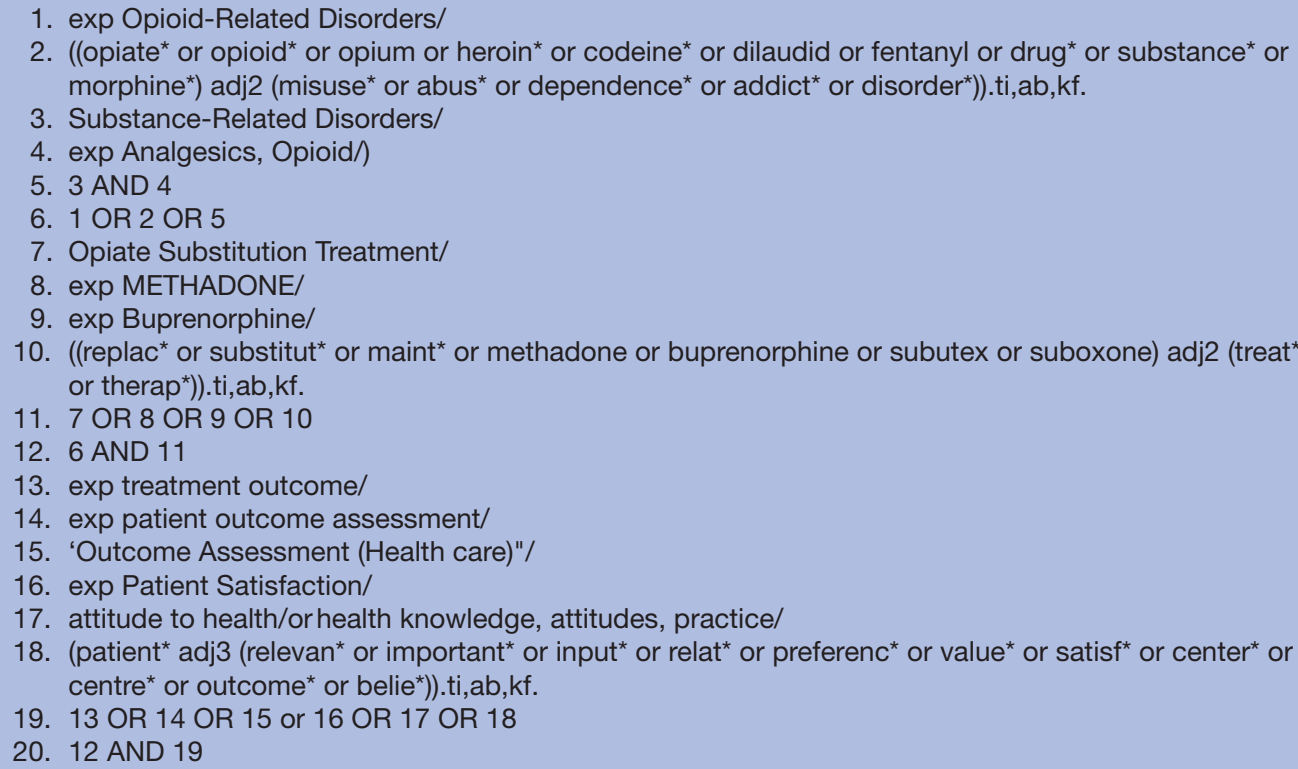 \\
\hline
\end{tabular}


experienced health sciences librarian will be consulted when devising the strategies and conducting the search in the identified databases. A broad search strategy will be employed to include titles, abstracts and keyword fields. No demographic or language constraints will be included in the search strategy, as OUD impacts individuals of all ages and nationalities. The searches will be limited to human studies.

\section{Data management}

An online platform, Google Forms, will be used in managing the articles and will allow the entire team to collaborate and easily access the articles. The team will be trained on how to use Google Forms. A calibration stage with 25 articles will take place to evaluate the level of agreement for the study form.

\section{Selection process}

For each research question, two reviewers will independently conduct title and abstract screening, and subsequently full text screening of those citations deemed potentially eligible. We will refer to the inclusion and exclusion criteria to assess study eligibility during screening. Disagreements between reviewers will be resolved by discussion to consensus, or by consulting a third author if it remains unresolved. We will assess agreement between reviewers and report the kappa statistic within our results. A flow diagram (figure 1) outlining the screening process, as well as a detailed table of included studies following the final screening stage will be included, in accordance with the Preferred Reporting Items for Systematic Reviews and Meta-Analyses guidelines. ${ }^{22}$ We will use ENDNOTE (V.x8) program to collect publication records, identify duplicates, find full text files and organise the articles for full text extraction.

\section{Data collection process}

For the first research question, full text data extraction forms will be constructed to include the following information: author, year of study, country, title of journal, number of participants, type of population (clinical, community or institutional), mean age and other demographic data of participants, study methodology (single arm, double arm or triple arm), type of MAT (methadone, buprenorphine, heroin etc), dose and duration of treatment, outcomes assessed in the study and the various measures used. The types of details for the measured outcomes will include what outcome is being measured, the definition of the outcome, how often it is measured and how it is measured.

The second research question will use a similar data extraction template, with the exception that outcomes assessed in the study that patients consider important for treatment will be extracted. Additionally, the second objective will include qualitative research. Themes based on patient-important outcomes will be extracted. The data extraction forms for both objectives will be pilot tested by two independent reviewers to determine their feasibility for this review. For both research questions, data will be extracted in duplicate to ensure accuracy.

\section{Risk of bias in individual studies}

Two reviewers will independently assess the methodological quality of eligible studies for this review. We will use the Cochrane Risk of Bias Tool to assess the risk of bias for RCTs. ${ }^{23}$ We will conduct subgroup analyses based on risk of bias assessment scores for studies scoring six points or higher on the Cochrane Risk of Bias Tool, which are standard cut-offs used in previous reviews. ${ }^{18}$ The Newcastle-Ottawa Scale will be used to assess the risk of bias for observational studies ${ }^{24}$ and will conduct subgroup analyses based on risk of bias assessment scores for studies scoring five or lower.

\section{Data synthesis}

Results from the first objective will be summarised qualitatively. This analysis will involve summary descriptions of all outcomes identified and used, the treatment domains of outcomes, summaries of the rationales provided for using said outcomes to measure treatment effectiveness. We will also summarise any psychometric properties associated with any outcome reported. Meanwhile, results from the second research question will be summarised qualitatively. Patient-important outcomes will be reviewed and grouped using a thematic analysis. Themes will emerge as the data are analysed, but some examples that may arise include drug-related outcomes, social outcomes, physical health outcomes and occupational outcomes.

\section{Confidence in cumulative evidence}

As we are purely reviewing what outcomes are reported and how these outcomes are measured for effectiveness of MAT, we will not need to determine strength of evidence using The Grading of Recommendations Assessment, Development and Evaluation. ${ }^{25}$

\section{Patient and public involvement}

As this is a protocol for a systematic review, there will be no patient or public involvement at this stage.

\section{ETHICS AND DISSEMINATION}

This study has planned and integrated a knowledge translation component. The primary end users of this information are clinicians, researchers and patients who use MAT. As such, we have chosen a knowledge users panel designing the review question and implementation. The goal is to broadly disseminate the synthesised information to improve the way treatment effectiveness is evaluated in MAT. We will collaborate primarily with the Canadian Addiction Treatment Centres (CATC), Canadian Centre for Substance Use and Addiction and addiction services in British Columbia (Dr Launette Rieb, addiction medicine) to disseminate the findings of this review by releasing summary reports and creating resources for knowledge users to access the information easily. We plan to share the results of our study through educational 
Primary search:

Pubmed/Medline, EMBASE, PsycINFO, CINAHL, Web of Science, Cochrane Library, Cochrane Clinical Trials Registry, and National Institutes for Health Clinical Trials Registry, W orld Health Organization International Clinical Trials Registry Platform

$\mathrm{n}=$

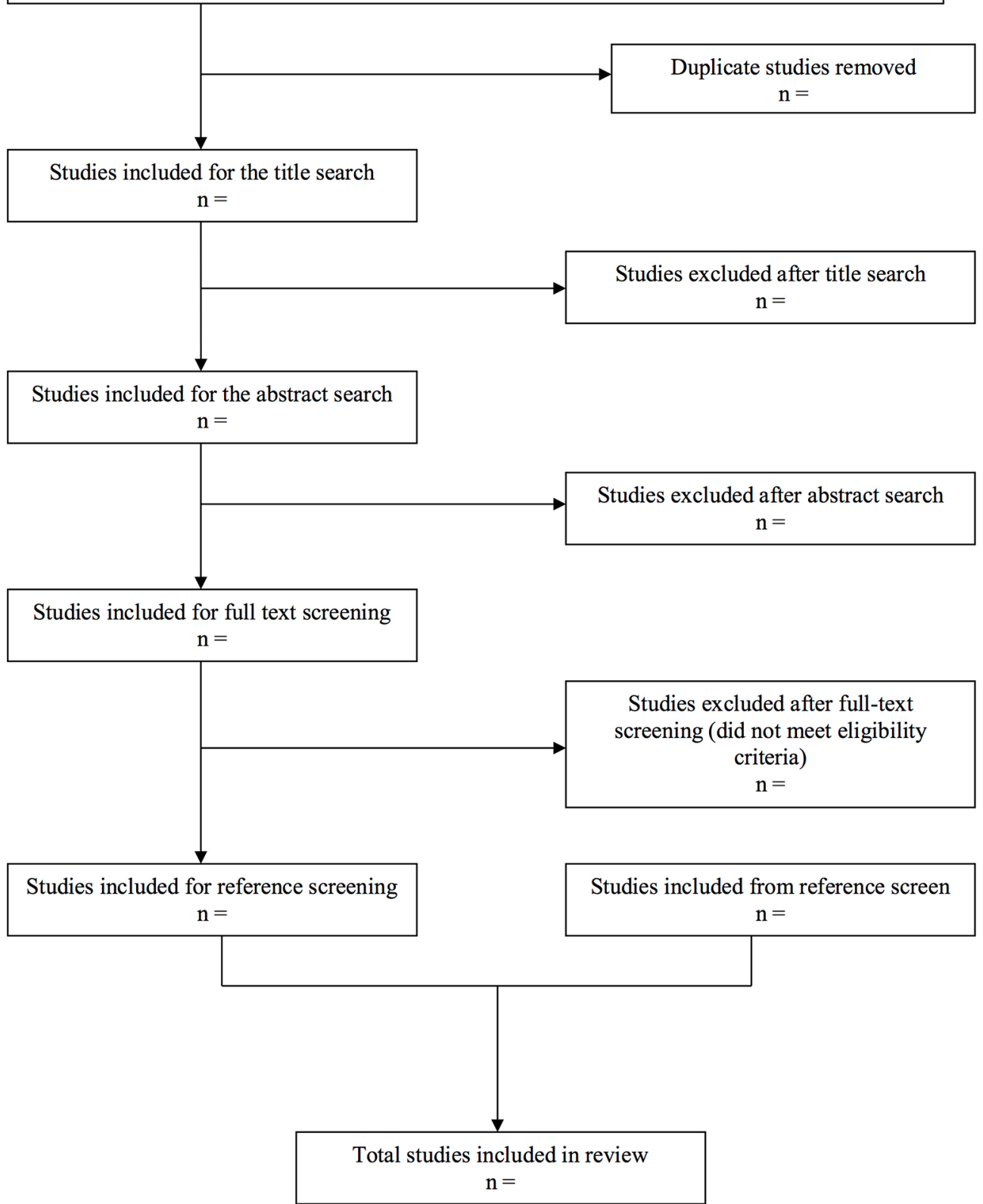

Figure 1 Flow diagram of included studies.

workshops for healthcare providers, such as primary care physicians, addiction specialists, service managers and researchers. We will also disseminate the information to the scientific community via peer-reviewed publications and conference presentations.

Author affiliations

${ }^{1}$ Medical Science Gradaute Program, McMaster University, Hamilton, Ontario, Canada

${ }^{2}$ Department of Psychiatry and Behavioural Neurosciences, McMaster University, Hamilton, Ontario, Canada

${ }^{3}$ Arts and Sciences, McMaster University, Hamilton, Ontario, Canada

${ }^{4}$ St. George's Hospital Medical School, University of London, London, UK
${ }^{5}$ Department of Health Research Methods, Evaluation, and Impact, McMaster University, Hamilton, Ontario, Canada

${ }^{6}$ Northern Ontario School of Medicine, Laurentian University, Sudbury, Ontario, Canada

${ }^{7}$ Canadian Addiction Treatment Centres, McMaster University, Markham, Ontario, Canada

${ }^{8}$ Department of Health Sciences: Health Science Library, McMaster University, Hamilton, Ontario, Canada

${ }^{9}$ Department of Medicine, Hamilton General Hospital, Hamilton, Ontario, Canada

${ }^{10}$ Rand Teed Consulting Services, Craven, Saskatchewan, Canada

${ }^{11}$ Department of Family Practice, Faculty of Medicine, University of British Columbia, Vancouver, British Columbia, Canada

${ }^{12}$ Ottawa Hospital Research Institute, Clinical Epidemiology Program, Ottawa, Ontario, Canada 
${ }^{13}$ Bruyère Research Institute, Ottawa, Ontario, Canada

${ }^{14}$ School of Epidemiology and Public Health, Faculty of Medicine, University of Ottawa, Ottawa, Ontario, Canada

${ }^{15}$ Department of Medicine, The Ottawa Hospital, Ottawa, Ontario, Canada

${ }^{16}$ School of Epidemiology, Public Health and Preventive Medicine, University of Ottawa, Ontario, Canada

${ }^{17}$ Institute for Work and Health, St. Michael's Hospital, Ottawa, Ontario, Canada

${ }^{18}$ Departments of Occupational Therapy and Medicine and the Clinical Epidemiology and Health Care Research Program, University of Toronto, Toronto, Ontario, Canada

${ }^{19}$ Faculty of Medicine, University of Ottawa, Ottawa, Ontario, Canada

${ }^{20}$ University of Ottawa Institute of Mental Health Research, Ottawa, Ontario, Canada

${ }^{21}$ Division of Pediatric Endocrinology, McMaster University, Hamilton, Ontario, Canada

22Department of Health Research Methods, Evidence and Impact, McMaster University, Hamilton, ontario, Canada

${ }^{23}$ Centre for Evaluation of Medicines, Programs for Assessment of Technology in

Health Research Institute, McMaster University, Hamilton, Ontario, Canada

Contributors NS: contributed to the conception and design of the study, development of data extraction forms, search strategy, manuscript writing and final review of the manuscript. HS: contributed to the methodological design, manuscript writing, critical revision and final review of the manuscript. BBD: contributed to the methodological design, critical revision and final review of the manuscript. $\mathrm{JH}$, DM, AW, LR, RT, PT, BH, DB, KC, BS, LM, DR, CS, RD, LT : contributed to the critical revision and final review of the manuscript. ZS: contributed to the conception and design of the study, critical revision and final approval of the manuscript. All authors read and approved the final manuscript.

Funding This research received no specific grant from any funding agency in the public, commercial or not-for-profit sectors. Dr Samaan is supported by grants from CIHR Award \#156306, Bridge CIHR Sponsor Award \#PJT-153429 and HAHSO Sponsor Award \#HAH-16-04.

Competing interests None declared.

Patient consent Not required.

Provenance and peer review Not commissioned; externally peer reviewed.

Open access This is an open access article distributed in accordance with the Creative Commons Attribution Non Commercial (CC BY-NC 4.0) license, which permits others to distribute, remix, adapt, build upon this work non-commercially, and license their derivative works on different terms, provided the original work is properly cited, appropriate credit is given, any changes made indicated, and the use is non-commercial. See: http://creativecommons.org/licenses/by-nc/4.0/.

\section{REFERENCES}

1. Sibbald B. Doctors 'responsibility on opioid crisis. CMAJ News. 2016 http://cmajnews.com/2016/11/18/doctors-responsibility-in-opioidcrisis-cmaj-109-5359/ (cited 2017 Jul 17).

2. Abuse $\mathrm{CC}$ of $\mathrm{S}$. The impact of substance use disorders on hospital use. hospital costs and impacts of substance use. $2014 \mathrm{http} / / / \mathrm{www}$. ccsa.ca/Eng/topics/Costs-of-Substance-Abuse-in-Canada/HospitalCosts-Impacts-of-Substance-Use/Pages/default.aspx

3. National Pain Center.; 2017.Busse J. The 2017 Canadian guideline for opioids for chronic non-cancer pain. Hamilton (ON): National Pain Center, 2017.
4. Abuse $\mathrm{CC}$ of $\mathrm{S}$. Collective challenge requires collective action. 2013;5-7:. http://www.ccsa.ca/Resource Library/CCSA-PrescriptionDrug-Strategy-news-release-2013-en.pdf

5. Canadian Institute for Health Information CC on SA. Preliminary rates of harm due to opioid poisoning, by province/territory. $2017 \mathrm{https} / / /$ www.cihi.ca/en/preliminary-rates-of-harm-due-to-opioid-poisoningby-provinceterritory-2017

6. Points K. Canadian Drug Summary. Can Cent Subst Abus, 2015:1-9.

7. Canada G of. National report: apparent opioid-related deaths. 2017 https://www.canada.ca/en/health-canada/services/substance-abuse/ prescription-drug-abuse/opioids/apparent-opioid-related-deaths. html

8. Fischer B, Rehm J, Brissette S, et al. Illicit opioid use in Canada: comparing social, health, and drug use characteristics of untreated users in five cities (OPICAN study). J Urban Health 2005;82:250-66.

9. Blackwell T. Critics question methadone usage as patients numbers soar in Canada: National Post, 2016.

10. Office of the Provincial Health Officer-British Columbia. British columbia oppoid substitution treatment system performance measures 2014/2015 - 2015/2016. 2017.

11. Bertschy G. Methadone maintenance treatment: an update. Eur Arch Psychiatry Clin Neurosci 1995;245:114-24.

12. Schuckit MA. Treatment of Opioid-Use Disorders. N Engl J Med 2016;375:357-68.

13. Bruneau J, Ahamad K, Goyer MÈ, et al. Management of opioid use disorders: a national clinical practice guideline. CMAJ 2018;190:E24 7-E257.

14. The College of Physicians \& Surgeons of Ontario. Methadone Maintenance Treatment. : Program Standards and Clinical Guidelines, 2011:2011: 1-148.

15. Mattick RP, Breen C, Kimber J, et al. Methadone maintenance therapy versus no opioid replacement therapy for opioid dependence. Cochrane Database Syst Rev 2009:CD002209.

16. Hedrich D, Alves $P$, Farrell M, et al. The effectiveness of opioid maintenance treatment in prison settings: a systematic review. Addiction 2012;107:501-17.

17. Li Y, Kantelip J-P, Schieveen PG-van, et al. Interindividual variability of methadone response. Mol Diagn Ther 2008;12:109-24.

18. Dennis BB, Roshanov PS, Naji L, et al. Opioid substitution and antagonist therapy trials exclude the common addiction patient: a systematic review and analysis of eligibility criteria. Trials 2015;16:475.

19. McLellan AT, Kushner H, Metzger D, et al. The fifth edition of the addiction severity index. J Subst Abuse Treat 1992;9:199-213.

20. Marsden J, Gossop M, Stewart D, et al. The maudsley addiction profile: development and user manual. Natl Addict Centre/Institute Psychiatry 1998:1-40.

21. Moher D, Shamseer L, Clarke M, et al. Preferred reporting items for systematic review and meta-analysis protocols (PRISMA-P) 2015 statement. Syst Rev 2015;4:1.

22. Moher D, Liberati A, Tetzlaff J, et al. Preferred reporting items for systematic reviews and meta-analyses: the PRISMA statement. PLoS Med 2009;6:e1000097.

23. Higgins JP, Altman DG, Gøtzsche PC, et al. The cochrane collaboration's tool for assessing risk of bias in randomised trials. BMJ 2011;343:d5928.

24. Wells GA, Shea B, O'connell D, et al. The Newcastle-Ottawa Scale (NOS) for assessing the quality if nonrandomized studies in metaanalyses. 2009 http//www ohri ca/programs/clinical_epidemiology/ oxford htm (cited 19 Oct 2009).

25. Guyatt $\mathrm{GH}$, Oxman $A D$, Schünemann HJ, et al. GRADE guidelines: a new series of articles in the journal of clinical epidemiology. J Clin Epidemiol 2011;64:380-2. 